\title{
Can DNA fragmentation of neat or swim-up spermatozoa be used to predict pregnancy following ICSI of fertile oocyte donors?
}

\author{
Jaime Gosálvez ${ }^{1}$, Pedro Caballero ${ }^{2}$, Carmen López-Fernández ${ }^{1}$, Leonor Ortega ${ }^{2}$, José Andrés Guijarro ${ }^{2}$, José Luís \\ Fernández $^{3}$, Stephen D Johnston ${ }^{4}$ and Rocío Nuñez-Calonge ${ }^{2}$
}

This study compared the potential of assessing sperm DNA fragmentation (SDF) from neat semen and the subsequent swim-up (SU) procedure to predict pregnancy when conducting ICSI of fertile donor oocytes. Infertile females $(n=81)$ were transferred embryos resulting from intracytoplasmic sperm injection (ICSI) of their partner's spermatozoa and proven donor oocytes. This model normalized the impact of female factor in putative sperm DNA repair. Semen was blindly assessed for SDF using Halosperm immediately following ejaculation (NS) and after swim-up at the time of ICSI fertilisation. There was a decrease in SDF values of the ejaculated semen sample following the swim-up protocol $(P=0.000)$. Interestingly, pregnancy could be equally predicted from SDF values derived from either neat or swim-up semen samples. Receiver operator curves and the derived Youden's indices determined SDF cutoff values for NS and SU of $24.8 \%$ and $17.5 \%$, respectively. Prediction of pregnancy from NS SDF had a sensitivity of $75 \%$ and a specificity of $69 \%$, whereas for SU SDF was $78 \%$ and $73 \%$, respectively. While increased levels of SDF negatively impact reproductive outcome, we have shown that a reduction in SDF following sperm selection using ICSI with proven donor oocytes is not mandatory for achieving pregnancy. This suggests that a certain level of DNA damage that is not detectable using current technologies could be impacting on the relative success of assisted reproductive technology (ART) procedures. Consequently, we propose a modification of the so called 'iceberg model' as a possible rationale for understanding the role of SDF in reproductive outcome.

Asian Journal of Andrology (2013) 15, 812-818; doi:10.1038/aja.2013.74; published online 14 October 2013

Keywords: assisted reproductive technology; egg donation; male factor; sperm DNA fragmentation

\section{INTRODUCTION}

Human classical seminal parameters that reliably predict the outcome of human assisted reproduction are controversial, ${ }^{1-4}$ but if available, would constitute a valuable tool for the reproductive physician. In this respect, the assessment of sperm DNA fragmentation (SDF) as a potential predictor of fertility is gaining increased acceptance $;^{5-7}$ in fact, we would suggest that this might particularly be the case for predictions of pregnancy based on intracytoplasmic sperm injection (ICSI), as this procedure requires no sperm motility and a single 'selected' spermatozoon, so that the effect of other sperm population parameters on reproductive outcome are largely negated or reduced. Under these conditions, it is possible that sperm DNA fragmentation is likely to be a better predictor of male factor infertility, especially that associated with compromised syngamy and early embryonic loss, ${ }^{8-11}$ although in other cases, this relationship is still not clear. ${ }^{12,13}$ When predictive values for reproductive outcome are compared using classical seminal parameters and sperm DNA damage assessment, the clinical validity of sperm DNA damage in intrauterine insemination (IUI) is higher than that obtained from sperm morphology alone; ${ }^{14}$ even so, there is still considerable heterogeneity among the different clinical contexts as presented in a meta-analysis recently conducted by Castilla et al. ${ }^{15}$ Reaching a consensus regarding a threshold level for SDF in terms of the 'Take home baby rate' has also been controversial; ${ }^{16}$ while a SDF rate ranging from $30 \%$ to $40 \%$ is considered by some as being predictive of poor fertility, ${ }^{6,8,17-19}$ there are some patients that possess a high level of SDF that are still capable of successful pregnancy, ${ }^{20}$ particularly when ICSI is used. Recently, Simon et $a .^{21}$ have reported that sperm DNA fragmentation was lower in couples achieving pregnancies after in vitro fertilisation (IVF) but not using ICSI after density gradient centrifugation for sperm selection.

Regarding the predictive role of DNA damage and pregnancy, the scenario is even more complicated when we examine the values obtained from neat semen samples versus selected samples. ${ }^{22}$ For example, in a meta-analysis performed by $\mathrm{Li}$ et al., ${ }^{23}$ there was no explicit reference as to the origin of the semen sample in terms of neat or selected at the time of SDF assessment, so that interpretation of data of this type is problematic. When sperm selection is performed after IVF, the effect of SDF on the pregnancy rate is relatively low but a meta-analysis by $\mathrm{Zini}^{24}$ has revealed that it was nevertheless apparent. Although this effect is not so clearly observed after sperm selection and

${ }^{1}$ Faculty of Biology, Autonomous University of Madrid, Cantoblanco, Madrid 28049, Spain; ${ }^{2}$ Clínica Tambre, Madrid 28002, Spain; ${ }^{3}$ GINIBIC-Complejo Hospitalario Universitario, A Coruña, A Coruña15006, Spain and ${ }^{4}$ School of Agriculture and Food Science, The University of Queensland, Gatton 4343, Australia

Correspondence: Professor J Gosálvez (jaime.gosalvez@uam.es)

Received: 11 February 2013; Revised: 12 March 2013; Accepted: 13 May 2013; Published online: 14 October 2013 
ICSI, ${ }^{14,25}$ there is still evidence showing that embryo quality may be affected. $^{26,27}$

Additionally, the sensitivity (true positive) and specificity (1: false positive) of using SDF as a predictor of pregnancy varies among different laboratories and is likely to be dependent on the type of ART employed and the varying skill of individual clinicians (see, for example, Tables 3 and 4 in Zini and Sigman ${ }^{28}$ and Zini ${ }^{29}$ ). This inconsistency is probably due to the fact that the genesis of SDF in the male is not a consequence of a single factor but of a series of inter-related events including defective spermatogenesis during chromatin remodelling, the interaction of spermatozoa with oxidative stress, exposure to bacterial infections, the presence of chromosomal abnormalities, ${ }^{30}$ constitutive genetic conditions, ${ }^{31,32}$ genomic modifications, such as telomere-shortening ${ }^{33}$ or environmental stress. ${ }^{34}$ The predictive value of SDF is also influenced by the quality and competence of the oocyte, and the capacity of the female gamete to repair sperm DNA damage following syngamy. ${ }^{35}$ While there is a scarcity of information available regarding the differential capacity of the human oocyte for sperm DNA repair, ${ }^{36,37}$ it is nevertheless still the case, that the oocyte contributes to at least $50 \%$ of the predictive value of a successful pregnancy. Consequently, the varying quality of the oocyte represents a major potential confounding variable when making fertility predictions based solely on SDF, so that the use of high-quality oocytes from proven donors, is likely to be a useful strategy for controlling female factor contribution; we propose that under such an experimental model, the influence of SDF should at the very least, be more easily detected.

The current study was conducted to determine the effect of SDF on the pregnancy rate of patients undergoing ICSI to fertilize oocytes from donors with proven fertility. Our aim was to investigate the predictive value of SDF from neat semen samples obtained and assessed directly after liquefaction with sperm of the same sample that had been processed for sperm selection using and swim-up and used in parallel for ICSI. The use of oocytes from proven donors was used in order to reduce female infertility as a confounding variable.

\section{MATERIALS AND METHODS}

\section{Oocytes and spermatozoa}

All patients in this study consented to their participation according to Spanish legislation and confidentiality for egg donation programs and following adherence to ethical standards. Eighty-one couples with severe female factor infertility (24-35 years old; mean \pm s.d.: 29.0 \pm 3.6 years) were incorporated into this study and all were processed in the Tambre Fertility Clinic, Madrid, Spain. In all cases, donor oocytes were from anonymous young women of proven fertility (22-25 years old, while spermatozoa for ICSI were provided by husbands or partners ranging in age from 26 to 52 years old (mean \pm s.d.: $31.6 \pm 6.6$ years). Semen samples were obtained following masturbation on the day of fertilisation, placed at room temperature for liquefaction and assessed for SDF. Prior to use in ICSI, the neat semen samples were processed using swim-up in modified Ham's F-10 basal mediumHEPES (Irvine Scientific, CA, USA). Following incubation for $30 \mathrm{~min}$, the supernatant was recovered for use in ICSI after a classical sperm selection using PVP medium (PolyVinyl Pyrrolidone; Origio, Firenze, Italy). SDF was conducted under two different scenarios using the same ejaculate: (i) neat or freshly ejaculated semen after liquefaction (NS) and (ii) spermatozoa selected using a swim-up procedure (SU). To avoid the impact of iatrogenic sperm damage, the NS was assessed for SDF immediately after ejaculate liquefaction. The processed sample was also assessed for SDF at the time of ICSI fertilization. This is an important aspect of the study, since DNA damage, far from being a static parameter, increases as the samples are incubated in vitro. This effect is observed both in fresh ${ }^{38}$ and in cryopreserved thawed samples. ${ }^{39}$ Consequently, the observed values at the time of ICSI fertilisation can be very different to those assessed before or after the time of fertilisation. Clinical pregnancy was confirmed when a gestational sac with foetal heartbeat was detected by ultrasound at 7 weeks of pregnancy.

\section{Assessment of DNA fragmentation}

SDF was assessed using the Halosperm G2 test (Halotech DNA, SL, Madrid, Spain) with minor modifications to the staining procedure. The sperm chromatin dispersion test is based on a two main steps (i) controlled DNA denaturation and (ii) controlled protamine removal; this gives rise to partially deproteinized nucleoids in which the DNA loops expand, forming halos of chromatin dispersion. Fragmented sperm nucleoids do not develop a dispersion halo or possess a halo with minimal dispersion; non-fragmented sperm show a significant halo of DNA dispersal (Figure 1). The use of mild acid DNA denaturation enhances protein removal, thus producing massive protamine depletion while leaving other proteins such as those forming the flagellum relatively intact (Figure 1). DNA haloes produced after the test were visualized by fluorescence microscopy using GelRed (Biotium, Hayward, CA, USA) to stain DNA. A Leica DMLA model motorized fluorescence microscope controlled with software for automatic scanning and image digitisation (Leica Microsystems, Barcelona, Spain) was used for SDF analysis. The microscope was equipped with a Leica EL6000 metal halide fluorescence light source, a charge coupled device (Leica DFC350 FX; Leica Microsystems) and Fluotar $\times 40$ objectives for routine scanning. The study was performed as a blind clinical trial. Three hundred spermatozoa were scored per sample and the proportion of sperm containing a fragmented DNA molecule calculated.

\section{Statistical analysis}

Non-parametric statistics for a non-Gaussian population, including pairwise comparisons using exact one-sided Wilcoxon and MannWhitney tests were performed. Correlation analysis was conducted using a Spearman's rank test. A receiver operating characteristic (ROC) curve and Youden's index were calculated to test the predictive value of SDF with respect to pregnancy. ROC analysis provides the

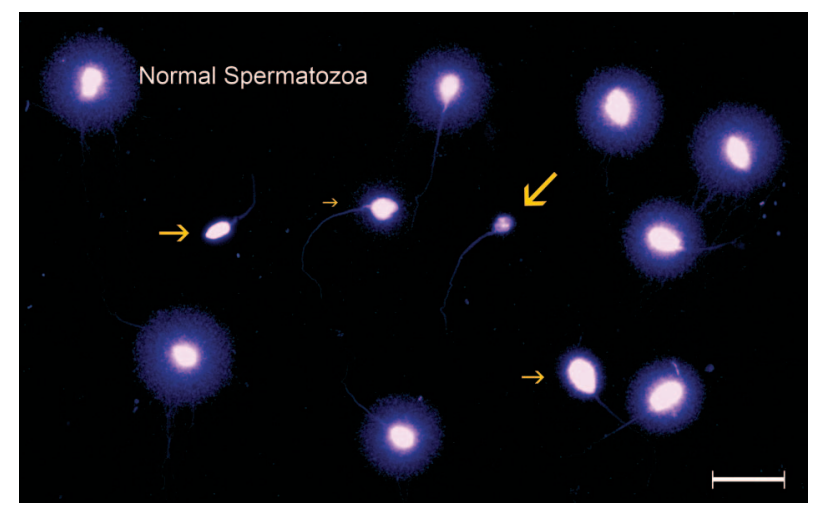

Figure 1 Sperm DNA fragmentation as visualized using Halosperm. Large haloes of chromatin dispersion around a compact core represent sperm showing an orthodox DNA molecule. Sperm presenting damage in the DNA are characterized by small or absent haloes of dispersed chromatin (green arrows). The size of the arrow indicates the extent of the DNA damage. Scale bar $=25 \mu \mathrm{m}$. 
most comprehensive description of diagnostic accuracy available to date because it estimates and reports all of the combinations of sensitivity and specificity that a diagnostic test is able to provide. ${ }^{40}$ ROC curves depict a test's ability to correctly identify 'true-positive' and 'true-negative' subjects for various test cutoff points, facilitating a comparison of the overall effectiveness, effectiveness at specified levels of false positives rates and optimal diagnostic ability. The Youden's index, $J$, is a measure of overall diagnostic effectiveness and is a function of sensitivity (probability of a truly diseased individual being diagnosed as such) and specificity (the probability of a truly healthy/ non-diseased individual being correctly diagnosed as non-diseased). ${ }^{41}$ The index ranges between 0 and 1 with a value of 1 indicating perfect diagnostic effectiveness and 0 indicating an ineffective test. In the majority of cases, there is an inverse relationship between sensitivity and specificity, so moving the 'cut point' increases one while reducing the other; $J$ occurs at the 'cut-point' that optimizes the biomarker's differentiating ability when equal weight is given to sensitivity and specificity. Conventionally, $J$ is found by evaluating sensitivity and specificity for all possible 'cut points', with the optimal 'cut-point' corresponding to $J$. Statistical analysis was performed using the Statistical Package for the Social Sciences 17 (SPSS Inc., Chicago, IL, USA); $P<0.05$ was defined as representing a significant difference.

\section{RESULTS}

\section{Descriptive statistics}

Eighty-one cycles of ICSI using donor oocyte and SU spermatozoa resulted in 49 successful pregnancies, or a pregnancy rate of $60.5 \%$. Table 1 shows the corresponding mean ( \pm s.d.) SDF values of the NS and SU sperm samples used in the ICSI procedure that subsequently resulted in pregnant and non-pregnant females.

\section{Efficacy of sperm DNA fragmentation via swim-up}

Pairwise comparisons using exact one-sided Wilcoxon tests revealed significant differences between the NS and SU SDF values observed within pregnant $(Z=-6.093 ; \quad P=0.000)$ and non-pregnant $(Z=-4.938$; $P=0.000$ ) groups (Table 1). To assess whether there was any difference with respect to the efficacy of the post-thaw sperm swim-up procedure on reducing SDF of sperm in either the pregnant and non-pregnant groups, an analysis of covariance of paired samples was performed comparing the change in SDF following swim-up in both groups; pregnancy was used as a cofactor in the model. The results of this analysis are shown in Figure 2 and revealed that the efficacy for SDF improvement was similar in both groups $(P=0.186)$ so that pregnancy was not linked to the efficiency in sperm improvement achieved after swim-up. Figure 3 shows the relationship between SDF values in NS and SU when data for pregnancy and non-pregnancy were pooled. Using a linear regression model, a significant correlation was found between SDF obtained in NS and SU samples $\left(R^{2}=84.8\right.$; ANOVA $F$ : $441.9 ; P<0.01$; where $\mathrm{NS}=2.94+1.34 \times x \mathrm{SU})$.

Table 1 Mean ( \pm s.d.) sperm DNA fragmentation (SDF) of neat (NS) and swim-up (SU) spermatozoa that resulted in pregnancy and nonpregnancy following ICSI of oocytes from proven donors

\begin{tabular}{lcc}
\hline & Pregnant $(\mathrm{n}=49)$ & Non-pregnant $(\mathrm{n}=32)$ \\
\hline Neat semen (NS) SDF & $25.3 \pm 14.5^{*}$ & $34.9 \pm 14.0^{* *}$ \\
Swim-up (SU) SDF & $16.6 \pm 9.1^{*}$ & $23.7 \pm 10.6^{* *}$ \\
\hline
\end{tabular}

Significant differences $(P<0.001)$ were obtained when NS and SU samples are compared within the pregnant group $(*)$ and non-pregnant-group $(* *)$. Data are expressed as mean \pm s.d.

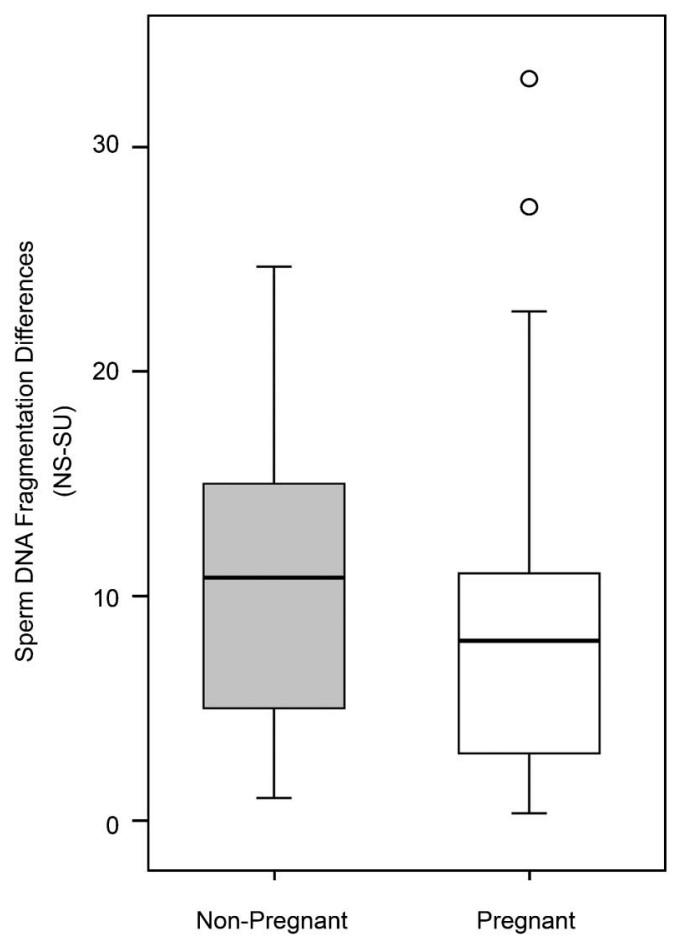

Figure 2 Box and whisker diagram showing the relative improvement in sperm DNA fragmentation following swim-up; sperm samples were grouped into patients where ICSI resulted in pregnancy $(n=49)$ or no pregnancy $(n=32) . I C S I$, intracytoplasmic sperm injection.

\section{Sperm DNA fragmentation and reproductive outcome}

SDF of the NS samples for pregnant and non-pregnant groups were statistically different from each other (Table 1; Mann-Whitney $U$ test $Z=-3.198 ; P=0.001$ ), as was the SDF of respective SU samples from the pregnant and non-pregnant groups (Table 1; Mann-Whitney test $Z=-3.198 ; P=0.001)$. The highest mean SDF value obtained in the experiment was that of the NS of the non-pregnant group, whereas the lowest level was that of the SU of the pregnant group (Table 1). It is interesting to highlight that the mean SDF NS value that resulted in pregnancy $(25.3 \pm 14.5)$ was not statistically different (Wilcoxon;

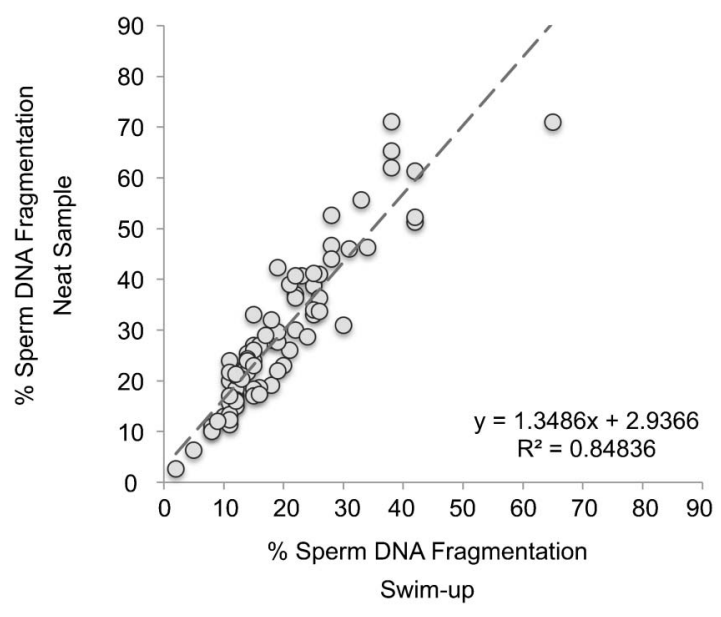

Figure 3 Correlation of \% sperm DNA fragmentation for neat semen samples and swim-up spermatozoa. Pooled data from pregnant $(n=49)$ and nonpregnant $(n=32)$ groups. 
$W=785.5 ; P \geqslant 0.05)$ to the mean SDF SU value of those patients that failed to produce a pregnancy $(23.7 \pm 10.6)$.

Since different values for SDF were obtained using the same ejaculate depending on whether the sample was swim-up or not and given the strong correlation between NS and SU (Figure 3), we tested for a possible correlation of SDF values before (NS) and after sperm selection (SU) but also took into account the success of pregnancy. A correlation analysis was performed for both pregnant and nonpregnant groups and a strong correlation was found in both groups (Spearman's rank test: $r=0.79 ; P<0.01$ for pregnant and $r=0.88$; $P<0.01$ for non-pregnant). To further explore this relationship, we tested the performance of our model by calculating the area under the ROC curve for NS and SU (Figure 4a). Using this statistical approximation, SDF assessment provided significant areas under the curve (AUC) for predicting pregnancy irrespective of whether the SDF for NS (AUC $=0.711, P<0.001)$ or SU $(\mathrm{AUC}=0.744, P<0.01)$ was used. Interestingly, not only the AUC but also the shape of the ROC curve depicted under different conditions (NS vs. SU) were similar and offered equivalent respective values for sensitivity and specificity (Figure 4a). The Youden's $J$ statistic, a single statistic that captures the performance of a diagnostic test, was 0.40 for the NS group resulting in a cutoff value for SDF of $26 \%$ (Figure $4 \mathbf{b}, 75 \%$ sensitivity and $65 \%$ of specificity). For the SU group, the value of the Youden's $J$ Index was 0.55 with a cutoff value based on SDF of $17.5 \%$ (Figure $4 c$,
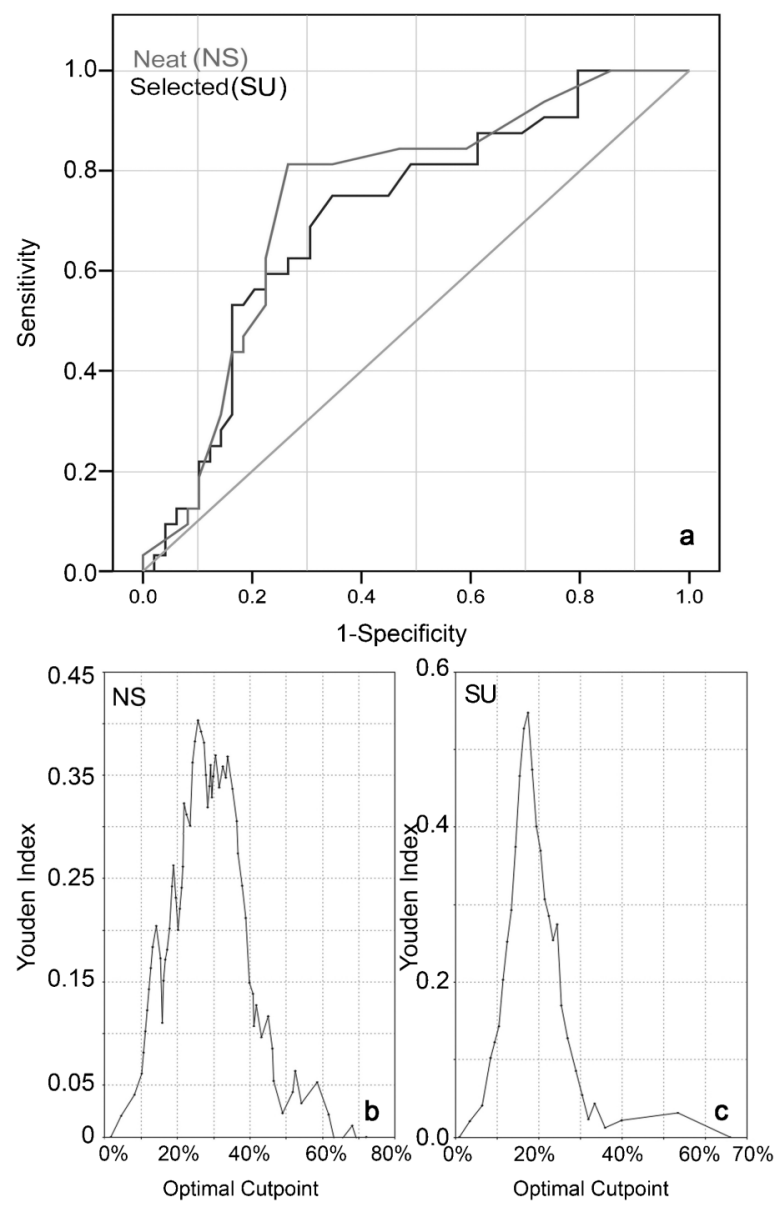

Figure 4 Receiver operating curves for neat (NS) and swim-up (SU) spermatozoa (a) and Youden's index plots of optimal cutoff points for NS (b) and SU (c) sperm DNA fragmentation data.
$81 \%$ sensitivity and $73 \%$ of specificity). In terms of prediction, an NS SDF value of lower than $26 \%$ accounted for a pregnancy rate of $80.0 \%$ (62.5\%-97.5\%; 4/20 cases), while an SDF higher than $17.5 \%$ shifted this percentage to $54.1 \%(41.6 \%-66.6 \%$; $28 / 61$ cases $)$; this gave an odds ratio of 3.39 with an approximate $95 \%$ confidence interval of 1.02-11.3. An SU SDF value of lower than $17.5 \%$ accounted for a pregnancy rate of $64.2 \%(52.7 \%-75.7 \% ; 24 / 67$ cases $)$, while in the group showing an SDF higher than $17.5 \%$, the percentage was $42.9 \%(17.0 \%-68.8 \% ; 8 / 14$ cases); this resulted in an odds ratio of 2.39 with an approximate $95 \%$ confidence interval (0.74-7.70).

\section{DISCUSSION}

This study revealed a significant decrease in the SDF values of the semen following the swim-up protocol; this reduction in SDF occurred in the semen of males irrespective of the reproductive outcome and indicates that a reduction in SDF is, therefore, not necessarily a strong indicator of a successful pregnancy when the sperm samples are used for ICSI. Nevertheless, this study has shown that pregnancy can be reasonably predicted from SDF values derived from either NS or SU semen samples and that in an experimental model that controls for both the quality of the donor oocyte and the SDF assessment at the time of fertilisation, Youden's index SDF 'cutoff' values for NS (26\%) and SU (17.5\%) produce an assumable predictive capacity for pregnancy. Prediction of pregnancy from NS SDF had sensitivity of $75 \%$ and specificity of $69 \%$, whereas the specificity and sensitivity based on SU SDF was 78\% and 73\%, respectively. Our findings highlight that there is no direct advantage, in terms of predictive value for pregnancy, when using SDF of NS. These results do not in any way imply that neat semen samples should be used for fertilisation or that the reproductive outcome would be similar using directly NS spermatozoa; rather, we propose that SDF assessment of the initial ejaculate has a certain level of predictive value according with the level of confidence obtained from the receiving operation curves and, therefore, could be used as a guideline by the practitioner on initial consultation with the patient. Given that female factor infertility was carefully controlled in this experiment by the use of high quality donor oocytes, our results also reinforce the importance of SDF as a contributing factor of male infertility.

\section{Sperm selection and sperm DNA damage}

The efficiency for sperm selection and its association with a decrease in SDF is a topic that has been studied from a range of perspectives ${ }^{42-45}$ but deserves further investigation, especially if we take into account possible collateral effects associated with the production of iatrogenic sperm damage via sperm handling. ${ }^{46}$ Some studies have reported the benefit of using selected spermatozoa to improve pregnancy ${ }^{47}$ and it is logical that the probability of selecting a sperm for ICSI free of DNA damage should increase if density gradient centrifugation or swim-up procedures are implemented. Congruent with this idea are those reports claiming that the predictive value of SDF is low when ICSI is used. ${ }^{14,15,21}$ It should be noted that while the incidence of SDF in the selected population may in fact decline after sperm selection, there is still a reasonable chance of accidently selecting one of the remaining underlying sub-population of DNA damaged spermatozoa that was not initially excluded in the selection procedure, i.e., a certain level of damaged spermatozoa still remains in the sample, even under the most stringent and rigorous conditions for sperm selection (see data in Zini et al. ${ }^{42}$ Gosálvez et al. ${ }^{38}$ and Enciso et al. ${ }^{43}$ ). By way of an example, if we analyse the data shown by Santiso et al. ${ }^{48}$ in Figure 1, in some cases and after a swim-up procedure, we are selecting sperm subpopulations 
containing shorter telomeres than those considered as normal. The selection of short telomeres could represent a problem for embryo development, since it produces a large heterozygosity for each chromosome pair with respect to the size of the telomeres. This undesired and undetectable fact may be lethal or at least as deleterious, as it could represent the presence of damaged DNA that was not removed after sperm selection.

We suggest that the swim-up methodology used here, while efficient at removing DNA damaged spermatozoa, did not remove sufficient spermatozoa harbouring apparently sublethal DNA damage in order to improve the capacity of the selected sample to increase reproductive outcome. In more severe male factor infertility, techniques such as PICSI (Physiological ICSI), IMSI (High Magnification Sperm inspection) or MACS (Magnetic Activated Cell Sorting) are potentially able to further refine the efficiency of sperm selection and thereby further increase the probability of a successful pregnancy. ${ }^{49-51}$

\section{Sperm DNA damage and threshold levels}

Another interesting finding from this investigation was the specific threshold levels for SDF offered in our predictive ICSI model based on the ROC analysis. As derived from the ROC and the Youden transformation, a neat semen sample with a SDF of $24.8 \%$ had the potential to predict pregnancy with sensitivity of $75 \%$ and a specificity of $69 \%$ (Figure 4). If the SDF increased by 10\%, then the value for sensitivity would decline to $60 \%$ and specificity increase to $73 \%$; a respective decline in SDF by $10 \%$ resulted in an increase for sensitivity to $75 \%$ and a decline of specificity to $66 \%$. For semen samples recovered after swim-up, and as derived from the ROC and the Youden transformation, the threshold value obtained for SDF was lower (17.5\%) than that obtained in neat semen (Figure 4); in this case, a sensitivity and specificity of $78 \%$ and $73 \%$ respectively were obtained. A $10 \%$ shift in SDF would result in a decrease of sensitivity to $67 \%$ but an increase in specificity to $75 \%$; if SDF declined by $10 \%$, then sensitivity and specificity changed to $78 \%$ and $64 \%$, respectively. If we compare these values with previously published data, there appears to be reasonable concordance; for example, it has been proposed that the SCSA technique can predict pregnancy failure when the sperm sample shows an SDF value $\geqslant 25 \%-30 \% .{ }^{18}$ Spano et al. ${ }^{8}$ and Giwercman et al. ${ }^{19}$ also showed that a level of sperm DNA damage $>20 \%$ was associated with decreased fertility and a reduced chance of natural conception. It should be remembered that these predictions have been made on the basis of studying ejaculated neat semen samples, so that these values may change if we use different techniques to determine SDF or if we compare different strategies for ART such as ICSI or IUI (see Table 3 in Zini and Libman ${ }^{5}$ and Bungum et al. $\left.{ }^{14}\right)$. Zini and coworkers $^{28,29}$ have also shown that for sensitivity and specificity, the confidence interval and the odds ratio will vary when the results from different laboratories are compared. This lack of standardisation in the use of SDF is perhaps not fully appreciated by reproductive clinicians but nevertheless is an extremely important contributing factor when making predictions about pregnancy. The incidence of sperm DNA damage has been shown to be inversely related to fertilisation rate following ICSI. ${ }^{52,53}$ Henkel et al. ${ }^{54}$ and Benchaib et al. ${ }^{55}$ have both reported its negative effect on pregnancy rate if sperm DNA damage was above $36 \%$ and $10 \%$, respectively. In IUI cycles, Bungum et al. ${ }^{14}$ suggested that SDF can be used as an independent predictor of fertility and proposed that a value of SDF lower than $30 \%$ resulted in approximately $19 \%$ children per cycle; they also noted that this proportion declined to $1.5 \%$, if SDF increased above $30 \%$. However, in other cases, no specific correlations were found between sperm
DNA damage as observed with the SCD test and pregnancy outcome after IUI. ${ }^{56}$ Interestingly in this study, sperm recovered by swim-up did not show a significant improvement in DNA integrity. Using also the sperm chromatin dispersion test and analysing a large cohort of patients attending for IVF or ICSI, Velez de la Calle et al. ${ }^{57}$ suggested a threshold sperm DNA fragmentation rate of $18 \%$, above which fragmentation rate was predictive of fertilisation rate. Evidently, the results of the comparison among these ART strategies could be biased since the ART strategies are very different (IUI, FIV-ICSI and egg donation-ICSI). A review of the literature revealed that the predictive value of pregnancy based on SDF appears to be more consistent for IVF/IUI than ICSI. ${ }^{14,15}$ The most consistent concept about the impact of sperm DNA damage on pregnancy is that semen prepared by density gradient centrifugation and used for ICSI, has a low power of predictive outcome. $^{21,22}$ The situation is quite confusing, and thus, for example, contrasting to those reports where no pregnancy is achieved following assisted reproduction above a sperm DNA fragmentation index of $28 \%,{ }^{58}$ other authors ${ }^{14}$ reported an IUI pregnancy in a man with a DFI of $34 \%$, and also pregnancies following IVF or ICSI with sperm DNA fragmentation indexes above $27 \%$. The negative impact of high levels of SDF on embryo quality as described by Borini et al. ${ }^{26}$ or derived from the information supplied by Zini et al., ${ }^{27}$ point to the possible fact that embryo development may be more significant altered in ICSI compared to IVF cycles.

In order to explain these observations, it must be remembered that the oocyte is also playing an important role in the final outcome of the embryo, primarily through its potential ability to repair sperm DNA. In the current experimental design, we assumed a high efficiency of DNA repair and homogeneity as the oocytes we used were all from proven donors; this allowed us to be more confident regarding our predictive SDF values for pregnancy. This is probably the explanation as to why we had reasonably similar predictive SDF values for pregnancy irrespective of whether neat (SDF: 24.8) or swim-up spermatozoa (SDF: 17.5$)$ were used. Where poor quality oocytes from infertile women are involved, the influence of SDF could be even more significant.

\section{The iceberg effect}

The unexpected lack of increased predictive power between neat and swim-up SDF reported in this study may be related to the phenomenon previously described by Evenson et al. ${ }^{59}$ and Álvarez ${ }^{60}$ as the 'iceberg effect' (Figure 5). This model proposed that the 'tip of the iceberg' corresponded to sperm DNA damage that is detectable using current available technologies i.e. sperm with massive DNA breakage. These spermatozoa are represented as the first level or the tip of the 'iceberg' in Figure $\mathbf{5}$ and correspond to easily detectable highly damaged sperm DNA. Following the swim-up procedure, those cells with detectable DNA damage are removed, but the sperm with undetectable SDF damage still remain hidden or cryptic within the population; these spermatozoa are found within the second level of the iceberg in Figure 5. It is possible that this subpopulation has not yet fully expressed itself in terms of SDF at the time of the analysis or selection and thereby represents what might be referred to as spermatozoa with a predisposition to SDF. These sperm are essentially cryptic in terms of SDF detection, waiting 'under the surface', ultimately to be detected, depending on the degree of damage imposed by ex vivo manipulation or iatrogenic damage prior to use in ART. We proposed that dynamic assessment of SDF by incubation of sperm in vitro ${ }^{38,39}$ would allow for the detection of this sub-population; ${ }^{61}$ this cryptic damage is essentially ignored by single assessments of DNA 


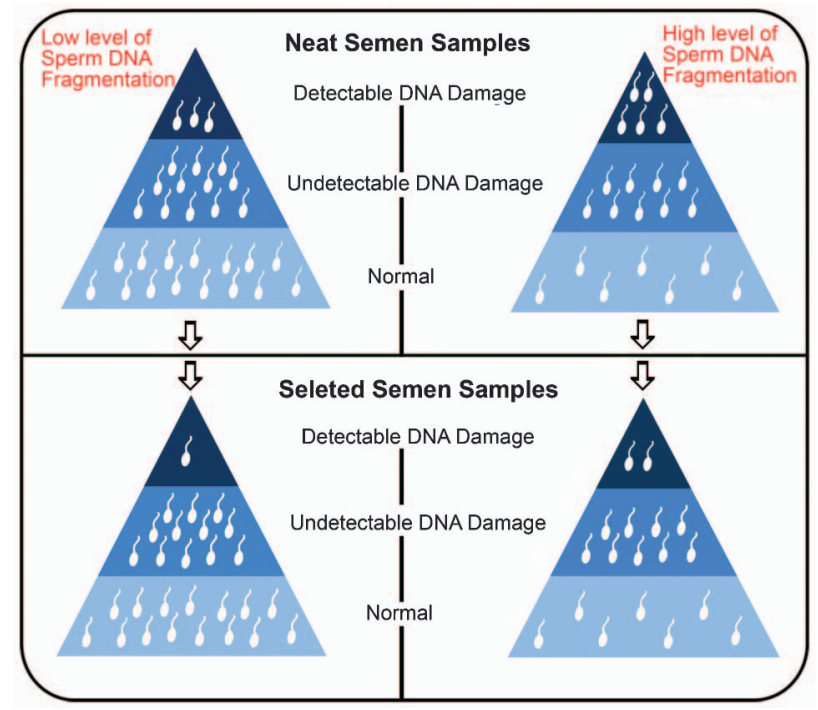

Figure 5 The iceberg effect of cryptic sperm DNA fragmentation adapted from the model originally proposed by Evenson et al. ${ }^{58}$

damage. It is possible that this cryptic subpopulation may contain sufficient DNA damage to prevent pregnancy, especially if the oocyte is not capable of DNA repair. The bottom layer of the 'iceberg' model (Figure 5) is representative of those spermatozoa that have highly stable DNA, but which under current methodologies cannot yet be physically selected. The primary difference between our model and that originally proposed by Evenson et al. ${ }^{59}$ is that we are suggesting that there is not always a strong positive correlation between spermatozoa found in the tip of the 'iceberg' and the proportion of spermatozoa in level 2 under the surface. For example, it is possible that a patient may have a low detectable level of SDF but a high underlying undetectable population of sperm with a predisposition for DNA damage. Alternatively, a patient may have a high detectable level of SDF but a low underlying subpopulation of sperm with a predisposition for DNA damage (Figure 5). The situation may also exist where a similar detectable level of SDF is present in two individuals, but differences in the underlying undetectable population are present.

Clearly, the amount, quality and distribution of DNA damage may vary among the different spermatozoa in the ejaculate, thus explaining the possibility of successful pregnancies despite a high SDF level. ${ }^{20} \mathrm{We}$ have already drawn attention to the efficiency of density gradient methodologies and swim-up techniques in eliminating spermatozoa containing double-strand DNA damage and sperm with highly damaged DNA, ${ }^{43}$ showing that density gradients are generally more efficient than swim-up at selecting spermatozoa that are free from single-strand DNA damage. ${ }^{43}$ Recently, Meseguer et al. ${ }^{35}$ showed that high-quality donated oocytes can potentially overcome the adverse influence of SDF on pregnancy. It is only when these type of issues and the compounding and interrelated influence of these factors are fully acknowledged that it is possible to make any sense of SDF as a predictor of reproductive success.

In conclusion, our experimental model appears to clarify, within the context of a proven egg donation program, why the sensitivity and specificity offered by the ROC curves are similar, despite different levels of SDF being observed between neat and swim-up spermatozoa. The assessment of SDF, when considered as a discrete value, is of importance to understanding reproductive outcome, but we suggest that it only has predictive value when analysed with respect to the specific ART in which it is used.

\section{AUTHOR CONTRIBUTIONS}

JG, PC and RNC were responsible for conception, experimental design and clinical follow up of the different patients. CLF and LO were in charge of the experimental content, data acquisition and data organisation. JLF, SDJ and JG were responsible for drafting the manuscript and intellectual content. JAG participated in the design of the study and performed the statistical analysis. All authors read and approved the final manuscript.

\section{COMPETING FINANCIAL INTERESTS}

The authors declare they have not any competing financial interests.

\section{ACKNOWLEDGMENTS}

This work was supported by Spanish Ministry of Science and Technology (MCYT: BFU2010-16738) and Fundación Clínica, Tambre.

1 Guzick DS, Overstreet JW, Factor-Litvek P, Brazil CK, Nakajima ST et al. Comparison of sperm morphology, motility, and concentration in fertile and infertile men. N Eng/ J Med 2001; 345: 1388-93.

2 Holt WV. Is quality assurance in semen analysis still really necessary? A spermatologist's viewpoint. Hum Reprod 2005; 20: 2983-6.

3 Lewis SEM. Is sperm evaluation useful in predicting human fertility? Reproduction 2007; 134: 31-40.

4 Schlegel PN. Evaluation of male infertility. Minerva Ginecol 2009; 61: 261-83.

5 Zini A, Libman J. Sperm DNA damage: clinical significance in the era of assisted reproduction. CMAJ 2006; 175: 495-500.

6 Bungum M, Bungum L, Giwercman A. Sperm chromatin structure assay (SCSA): a tool in diagnosis and treatment of infertility. Asian J Androl 2011; 13: 69-75.

7 Gosálvez J, López-Fernández C, Fernández JL. Sperm Chromatin Dispersion (SCD) test: technical aspects and clinical applications. In: Zini A, Agarwal A, editors. Sperm DNA Damage: Biological and Clinical Applications in Male Infertility and Assisted Reproduction. New York: Springer-Verlag; 2011. p151-70.

8 Spano M, Bonde JP, Hjollund HI, Kolstad HA, Cordelli E et al. Sperm chromatin damage impairs human fertility. The Danish first pregnancy planner study team. Fertil Steril 2000; 73: 43-50.

9 Henkel R, Hajimohammad M, Stalf T, Hoogendijk C, Mehnert C et al. Influence of deoxyribonucleic acid damage on fertilization and pregnancy. Fertil Steril 2004; 81: 965-72.

10 Benchaib M, Lornage J, Mazoyer C, Lejeune H, Salle B et al. Sperm deoxyribonucleic acid fragmentation as a prognostic indicator of assisted reproductive technology outcome. Fertil Steril 2007; 87: 93-100.

11 Bakos HW, Thompson JG, Feil D, Lane M. Sperm DNA damage is associated with assisted reproductive technology pregnancy. Int J Androl 2008; 31: 518-26.

12 Gandini L, Lombardo F, Paoli D, Caruso F, Eleuteri P et al. Full-term pregnancies achieved with ICSI despite high levels of sperm chromatin damage. Hum Reprod 2004: 19: 1409-17.

13 Virro MR, Kjersten L, Larson-Cook KL, Evenson DP. Sperm chromatin structure assay (SCSA) parameters are related to fertilization, blastocyst development, and ongoing pregnancy in in vitro fertilization and intracytoplamic sperm injection cycles. Fertil Steril 2004; 81: 1289-95.

14 Bungum M, Humaidan P, Spano M, Jepson K, Bungum L et al. The predictive value of sperm chromatin structure assay (SCSA) parameters for the outcome of intrauterine insemination, IVF and ICSI. Hum Reprod 2004; 19: 1401-8.

15 Castilla JA, Zamora S, Gonzalvo MC, Luna del Castillo JD, Roldan-Nofuentes JA et al. Sperm chromatin structure assay and classical semen parameters: systematic review. RBM Online 2010; 20: 114-24.

16 Zini A, Libman J. Sperm DNA damage: importance in the era of assisted reproduction. Curr Opin Urol 2006; 16: 428-34.

17 Evenson DP, Jost LK, Marshal D, Zinaman MJ, Clegg E et al. Utility of the sperm chromatin structure assay as a diagnostic and prognostic tool in the human fertility clinic. Hum Reprod 1999; 14: 1039-49.

18 Evenson DP, Wixon R. Data analysis of two in vivo fertility studies using Sperm Chromatin Structure Assay-derived DNA fragmentation index vs pregnancy outcome. Fertil Steril 2008; 90: 1229-31.

19 Giwercman A, Lindstedt L, Larsson M, Bungum M, Spano M et al. Sperm chromatin structure assay as an independent predictor of fertility in vivo: a case-control study. Int $J$ Androl 2010; 33: e221-7.

20 Payne JF, Raburn DJ, Couchman GM, Price TM, Jamison MG et al. Redefining the relationship between sperm deoxyribonucleic acid fragmentation as measured by the sperm chromatin structure assay and outcomes of assisted reproductive techniques. Fertil Steril 2005; 84: 356-64.

21 Simon L, Castillo J, Oliva R, Lewis SE. Relationships between human sperm protamines, DNA damage and assisted reproduction outcomes. RBM Online 2011; 23: $724-34$ 
22 Bungum M, Spano M, Humaidan P, Eleuteri P, Rescia M et al. Sperm chromatin structure assay parameters measured after density gradient centrifugation are not predictive for the outcome of ART. Hum Reprod 2008; 23: 4-10.

23 Li Z, Wang L, Cai J, Huang H. Correlation of sperm DNA damage with IVF and ICSI outcomes: a systematic review and meta-analysis. J Assist Reprod Genet 2006; 23. 367-76.

24 Zini A. Are sperm chromatin and DNA defects relevant in the clinic? Sys Biol Reprod Med 2011; 57: 78-85.

25 Bungum M, Humaidan P, Axmon A, Spano M, Bungum L et al. Sperm DNA integrity assessment in prediction of assisted reproduction technology outcome. Hum Reprod 2007; 22: 174-9.

26 Borini A, Tarozzi N, Bizzaro D, Bonu MA, Fava L et al. Sperm DNA fragmentation: paternal effect on early post-implantation embryo development in ART. Hum Reprod 2006; 21 : 2876-81.

27 Zini A, Meriano J, Kader K, Jarvi K, Laskin CA et al. Potential adverse effect of sperm DNA damage on embryo quality after ICSI. Hum Reprod 2005; 20: 3476-80.

28 Zini A, Sigman M. Are tests of sperm DNA damage clinically useful? Pros and cons. $J$ Androl 2009; 30: 219-29.

29 Zini A. Clinical utility of sperm DNA integrity tests. In: Zini A, Agarwal A, editors. Sperm DNA damage: Biological and Clinical Applications in Male Infertility and Assisted Reproduction. New York: Springer-Verlag; 2011. p499-504.

30 Fernández JL, Vélez de la Calle JF, Tamayo M, Cajigal D, Agarwal A et al. Sperm DNA integrity and male infertility: current perspectives. Arch Med Sci 2009; 5: S55-62.

31 Muriel L, Goyanes V, Segrelle E, Gosálvez J, Alvarez J et al. Increased aneuploidy rate in sperm with fragmented DNA as determined by the sperm chromatin dispersion (SCD) test and Fish analysis. J Androl 2007; 28: 38-49.

32 García-Peiró A, Martínez-Heredia J, Oliver-Bonet M, Abad C, Amengua JM et al. Protamine P1/P2 ratio correlates with dynamic aspects of DNA fragmentation in human sperm. Fertil Steril 2011; 95: 105-9.

33 Rodríguez S, Goyanes V, Segrelles E, Blasco M, Gosálvez J et al. Critically short telomeres are associated with sperm DNA fragmentation. Fertil Steril 2005; 84: 843-5.

34 Hansen C, Luben TJ, Sacks JD, Olshan A, Jeffay S et al. The effect of ambient air pollution on sperm quality. Environ Health Perspect 2010; 118: 203-9.

35 Meseguer M, Santiso R, Garrido N, García-Herrero S, Remohí J et al. Effect of sperm DNA fragmentation on pregnancy outcome depends on oocyte quality. Fertil Steril 2011; 95: 124-8.

36 Ashwood-Smith MJ, Edwards RG. DNA repair by oocytes. Mol Hum Reprod 1996; 2 : $46-51$.

37 Menezo Y Jr,Russo GL, Tosti E, Mouatassim SE, Benkhalifa M. Expression profile of genes coding for DNA repair in human oocytes using pangenomic microarrays, with a special focus on ROS linked decays. J Assist Reprod Genet 2007; 11: 513-20.

38 Gosálvez J, de la Torre J, López-Fernández C, Pérez-Gutiérrez L, Ortega L et al. DNA fragmentation dynamics in fresh versus frozen thawed plus gradient-isolated human spermatozoa. Syst Biol Reprod Med 2010; 56: 27-36.

39 Gosálvez J, Cortés-Gutierrez E, López-Fernández C, Fernández JL, Caballero P et al. Sperm DNA fragmentation dynamics in fertile donors. Fertil Steril 2009; 92: 170-3.

40 Metz CE. Receiver operating Characteristic analysis: a tool for the quantitative evaluation of observer performance and imaging systems. J Am Coll Radiol 2006; 6: 413-22.

41 Fluss R, Faraggi D, Reiser B. Estimation of the Youden Index and its associated cut off point. Biometrical J 2005; 47: 458-72.

42 Zini A, Finellia A, Phanga D, Jarvia K. Influence of semen processing technique on human sperm DNA integrity. Urology 2000; 56: 1081-4.
43 Enciso M, Iglesias M, Galán I, Sarasa J, Gosálvez A et al. The ability of sperm selection techniques to remove single- or double-strand DNA damage. Asian J Androl2011; 13: 764-8.

44 Ng FL, Liu DY, Baker HW. Comparison of Percoll, mini-Percoll and swim-up methods for sperm preparation from abnormal semen samples. Hum Reprod 1992; 7: 261-6.

45 Sakkas D, Manicardi GC, Tomlinson M, Mandrioli M, Bizzaro D et al. The use of two density gradient centrifugation techniques and the swim-up method to separate spermatozoa with chromatin and nuclear DNA anomalies. Hum Reprod 2000; 15: 1112-6.

46 Mortimer D. Sperm preparation techniques and iatrogenic failures of in-vitro fertilization. Hum Reprod 1991; 6: 173-6.

47 Tampaichitr N, Agulnik A, Seibel M, Taymor M. Comparison of the in vitro fertilization rate by human sperm capacitated by multiple-tube swim-up and Percoll gradient centrifugation. J In Vitro Fert Embryo Transf 1988; 5: 119-22.

48 Santiso R, Tamayo M, Gosálvez J, Meseguer M,Garrido N et al. Swim-up procedure selects spermatozoa with longer telomere length. Mut Res 2010; 688: 88-90.

49 Huszar G, Ozenci CC, Cayli S, Zavaczki Z, Hansch E et al. Hyaluronic acid binding by human sperm indicates cellular maturity, viability, and unreacted acrosomal status. Fertil Steril 2003; 79: 1616-24.

50 Eltes F, Yaari S, Katz N, Barr I, Fishman A et al. The morphological normalcy of the sperm nucleus and pregnancy rate of intracytoplasmic injection with morphologically selected sperm. Hum Reprod 2005; 20: 185-90.

51 Said TM, Agarwal A, Grunewald S, Rasch M, Glander HJ et al. Evaluation of sperm recovery following annexin $\mathrm{V}$ magnetic-activated cell sorting separation. RBM Online 2006; 13: 336-9.

52 Sun JG, Jurisicova A, Casper RF. Detection of deoxyribonucleic acid fragmentation in human sperm: correlation with fertilization in vitro. Biol Reprod 1997; 56: 602-7.

53 Lopes S, Sun JG, Jurisicova A, Meriano J, Casper RF. Sperm deoxyribonucleic acid fragmentation is increased in poor-quality semen samples and correlates with failed fertilization in intracytoplasmic sperm injection. Fertil Steril 1998; 69: 528-32.

54 Henkel R, Kierspel E, Stalf T, Mehnert C, Menkveld R. Effect of reactive oxygen species produced by spermatozoa and leukocytes on sperm functions in non leukocytospermic patients. Fertil Steril 2005; 83: 635-42.

55 Benchaib M, Braun V, Lornage J, Hadj S, Salle B et al. Sperm DNA fragmentation decreases the pregnancy rate in an assisted reproductive technique. Hum Reprod 2003; 18: 1023-8.

56 Muriel L, Meseguer M, Fernández JL, Álvarez J, Remohí J et al. Value of the sperm chromatin dispersion test in predicting pregnancy outcome in intrauterine insemination: a blind prospective study. Hum Reprod 2006; 21: 738-44.

57 Velez de la Calle JF, Muller A, Walschaerts M, Clavere JL, Jimenez C et al. Sperm deoxyribonucleic acid fragmentation as assessed by the sperm chromatin dispersion test in assisted reproductive technology programs: results of a large prospective multicenter study. Fertil Steril 2008; 90: 1792-9.

58 Evenson D, Jost L. Sperm chromatin structure assay is useful for fertility assessment. Methods Cell Sci 2000; 22: 169-89.

59 Evenson DP, Larson KJ, Jost LK. Sperm Chromatin Structure Assay: its clinical use for detecting sperm DNA fragmentation in male infertility and comparisons with other techniques. J Androl 2002; 23: 25-43.

60 Álvarez JG. The predictive value of sperm chromatin structure assay. Hum Reprod 2005; 20: 2365-7.

61 Santiso R, Tamayo M, Gosálvez J, Johnston S, Mariño A et al. DNA fragmentation dynamics allows the assessment of cryptic sperm damage in human: evaluation of exposure to ionizing radiation, hyperthermia, acidic $\mathrm{pH}$ and nitric oxide. Mutat Res 2012; 734: 41-9. 